\title{
BANDWIDTH-PERFORMANCE TRADEOFFS FOR A TRANSMISSION WITH CONCURRENT SIGNALS
}

\author{
Aminata A. Garba \\ Dept. of Electrical and Computer Engineering, Carnegie Mellon University \\ aminata@ece. cmu.edu
}

\begin{abstract}
We consider a bandwidth-efficient transmission scheme, where two signals are sent concurrently. The BER and the achievable minimum distances for the signals' constellations at the receivers are derived as functions of the signals' energies and their input probability distributions. Finally, trade-offs between bandwidth, signals' energies and achievable performances are discussed.
\end{abstract}

\section{KEYWORDS}

Bandwidth efficiency, Energy, Interference, Minimum Distance, BER Performance.

\section{INTRODUCTION}

The demand of bandwidth is largely increasing due to the explosion of smart devices and bandwidth-greedy applications. This has led to the development of several bandwidth efficient technologies such as Code Division Multiplexing Access, Multiple-Input, Multiple-Output (MIMO), ultra wideband (UWB), cognitive radio (CR) technologies. Nonetheless, a major limitation of bandwidth efficient technologies is the interference caused by simultaneous transmissions [1]-[5]. It is then important to analyze the impact of simultaneous signal transmissions on the system performance. In [6], symbol error probability is analyzed for a multiuser detector using successive cancellation, when signals differentiated by their powers are transmitted simultaneously.

In this paper, we consider simultaneous transmission of two signals and analyze the impact on the system performance. We present performance analysis and compare the resulting performance with the achievable performance if the signals were sent separately over different channels. We also discuss impact of interference and trade-offs between performance and bandwidth efficiency.

\section{SYSTEM MODEL \& ASSUMPTIONS}

We consider a communication scheme, where two signals are transmitted concurrently and independently (i.e., without collaboration). For example, this scheme can model a cognitive transmission where the cognitive signal interferes with the primary signal, either in a controlled interference scenario or because the cognitive user sensed an idle channel while the primary user was actually transmitting, due for example to challenges related to sensing in cognitive transmissions [7]. It can also model a different transmission scheme whereby; users with no or Jan Zizka et al. (Eds) : CCSEIT, MoWiN, IT, AIAP, ICBB - 2015 
small cognition capabilities can transmit simultaneously without time, frequency or code division multiplexing, thus interfering with each other.

The two signals, which may first be encoded using forward error correcting code, are modulated using BPSK modulation and sent concurrently over an AWGN channel.

Under these assumptions, the received symbol can be written as follows:

$$
\mathrm{Y}=\mathrm{X}_{1}+\mathrm{X}_{2}+\mathrm{n}
$$

where $X_{1}$ and $X_{2}$ are the (encoded and) modulated symbols from the two sources and $n$ is additive white Gaussian noise (AWGN) with zero mean and variance $\sigma^{2}$ that corrupts the received information. Furthermore, we assume that X1 has energy E1 and takes values in the input alphabet $A=\{-a, a\}$ with probability $P$ and (1-P) and $X 2$, has energy $E 2$ and takes values in its input alphabet $\mathrm{B}=\{-\mathrm{b}, \mathrm{b}\}$ with probabilities $\mathrm{P}$ and $(1-\mathrm{P})$. Finally, $\mathrm{a}, \mathrm{b}$ are assumed non-negative valued constants known to the receivers with ( for simplicity) $a \geq b$, i.e., $E_{1} \geq E_{2}$. ( The results for the case where $\mathrm{E}_{1}<\mathrm{E}_{2}$ can be derived from the considered case.)

At the receiver, minimum-distance symbol-by-symbol detection is used, i.e., a received symbol is detected as the closest point in the signal constellation. Finally, the received symbol $\mathrm{Y}$ is used to make decisions on the sent symbols $\mathrm{X}_{1}$ and $\mathrm{X}_{2}$ at both receivers respectively.

We refer to the minimum distance of a signal constellation at a given receiver, as the minimum of the distances between any two points of the signal constellation.

Furthermore, throughout this paper, $\hat{d}_{\min 1}$ and $\hat{d}_{\min 2}$ define the minimum distances for the signal constellations at the receivers 1 and 2 respectively, if each signal was sent separately (scenario 1 ). On the other hand, $\mathrm{d}_{\min 1}$ and $\mathrm{d}_{\min 2}$ represent the minimum distances for received constellations at receivers 1 and 2, when both symbols are sent concurrently (scenario 2). For both scenarios, we consider the same transmission conditions (symbols' energies, channel conditions, SNR).

Finally, since in AWGN channels, the performance strongly depends on the minimum distance of the received signal constellation, we next analyze the minimum distances $d_{\min 1}$ and $d_{\min 2}$ for the signals' constellations at the receivers when the 2 symbols are transmitted concurrently.

\section{Propositions \& LEMmas}

\section{A. Proposition 1}

The achievable minimum distance for a transmission with two signals sent concurrently and independently is at most the difference of the achievable minimum distances of the constellations if the signals were sent separately under the same conditions.

In particular, the minimum distance $\mathrm{d}_{\min 1}$ and $\mathrm{d}_{\min 2}$ of the received signals constellations, are respectively

$$
\begin{aligned}
\mathrm{d}_{\min 1} & =\hat{d}_{\min 1}-\hat{d}_{\min 2}, \\
\mathrm{~d}_{\min 2} & =\min \left(\hat{d}_{\min 2}, \hat{d}_{\min 1}-\hat{d}_{\min 2}\right)
\end{aligned}
$$




\section{Proof}

We assume BPSK modulated symbols at the transmitters and minimum-distance detection at the receivers. Assuming that the first signal uses input alphabet $\{-\mathrm{a}, \mathrm{a}\}$ with probability $\mathrm{P}$ and (1-P) and the second signal uses $\{-\mathrm{b}, \mathrm{b}\}$ with probability $\mathrm{P}$ and $(1-\mathrm{P})$, the minimum distances of the received constellations are given by $\hat{d}_{\min 1}=2 \mathrm{a}, \hat{d}_{\min 2}=2 \mathrm{~b}$ is each signal is transmitted separately (scenario 1).

If the two signals were sent concurrently (scenario 2) and assuming a minimum-distance detector, the decision regions are shown in Figure 1. (Note that the minimum distance detection is equivalent to the ML detection for AWGN channels.)

Using the decision regions illustrated in Figure 1, the minimum distances of the signal constellations at the receivers are given by:

$$
\begin{aligned}
d_{\min } & =(a-b)-(-a+b)=2(a-b)=\hat{d}_{\min 1}-\hat{d}_{\min 2}, \\
d_{\min _{2}} & =\min ((a-b)-(-a+b), 2(a-b))=\min \left(2 b, d_{\min 1}\right) \\
& =\min \left(\hat{d}_{\min 2},\left(\hat{d}_{\min 1}-\hat{d}_{\min 2}\right)\right) .
\end{aligned}
$$

\section{B. Proposition 2}

1. If $\mathrm{E}_{1} \neq \mathrm{E}_{2}$, then the minimum distance $\mathrm{d}_{\min 1}$ and $\mathrm{d}_{\min 2}$ of the signals' constellations at receivers 1 and 2 , are respectively

$$
\begin{aligned}
d_{\min }= & \hat{d}_{\min 1}-\hat{d}_{\min 2}=2\left(\sqrt{E_{1}}-\sqrt{E_{2}}\right) \\
d_{\min 2} & =\min \left(\hat{d}_{\min 2}, \hat{d}_{\min 1}-\hat{d}_{\min 2}\right) \\
& = \begin{cases}\hat{d}_{\min 2}=2 \sqrt{E_{2}} & \text { if } E_{1} \geq 4 E_{2} \\
\hat{d}_{\min 1}-\hat{d}_{\min 2}=2\left(\sqrt{E_{1}}-\sqrt{E_{2}}\right) & \text { if } E_{1}<4 E_{2}\end{cases}
\end{aligned}
$$

2. If the signals have the same energy $\left(\mathrm{E}_{2}=\mathrm{E}_{1}=\mathrm{E}\right)$, the minimum distance is zero for both constellations): $\quad d_{\text {min }_{1}}=d_{\text {min }_{2}}=0$.

\section{Proof}

Part 1 of the proposition results from equation (3) using the average symbol energy given as follows:

$$
\begin{gathered}
E_{1}=\text { mean }\left(X_{1}^{2}\right)=a^{2} P+a^{2}(1-P)=a^{2} \\
\Rightarrow a=\sqrt{E_{1}} ; \text { similarly } b=\sqrt{E_{2}}
\end{gathered}
$$

Part 2 of the proposition follows from part 1 when $\mathrm{E}_{1}=\mathrm{E}_{2}$. 


\section{Proposition 3}

When 2 signals are sent concurrently as described in section II, the BER performance for the strongest signal can be determined as follows:

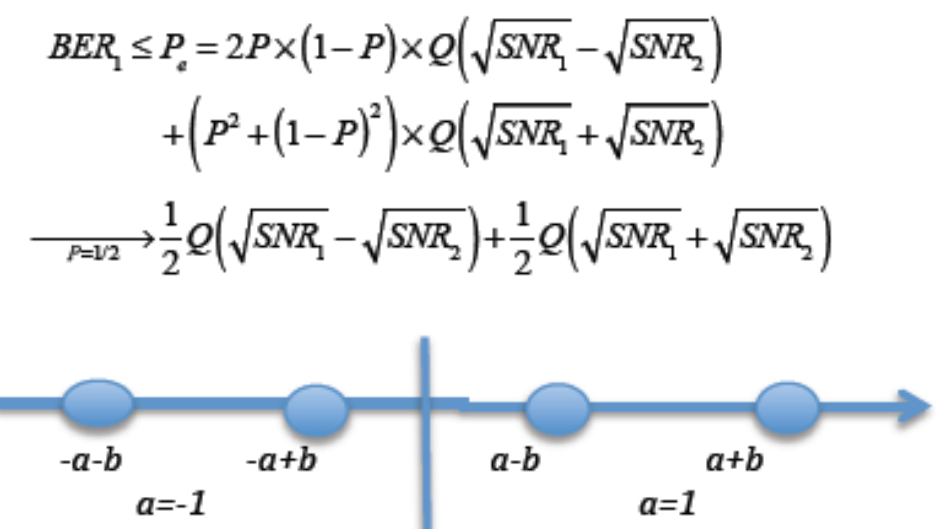

Signal space and decision region at the first receiver

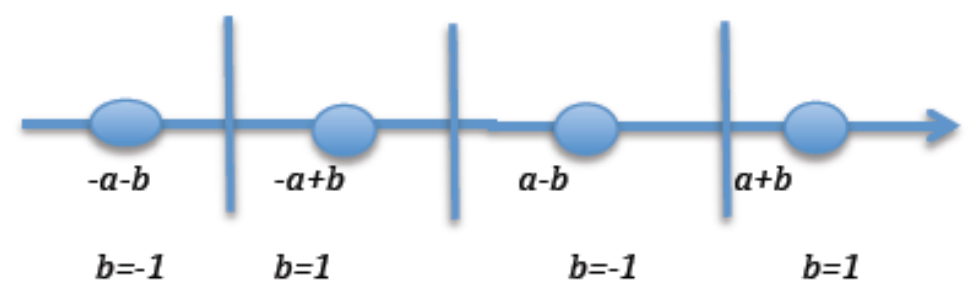

Signal space and decision regions for the second receiver

Figure 1: Signal space and decision regions

where $[\mathrm{P},(1-\mathrm{P})]$ is the probability input distribution for the considered binary signaling, $\mathrm{SNR}_{1}$, $\mathrm{SNR}_{2}$ are the signal to noise ratios of the individual signals and $\mathrm{Q}(\mathrm{x})$ is the standard $\mathrm{Q}$ function defined by $\mathrm{Q}(\mathrm{x})=\operatorname{Pr}(\mathrm{X}>\mathrm{x})=\operatorname{Pr}(\mathrm{X}<-\mathrm{x})$ when $\mathrm{X}$ is zero mean, variance 1 , Gaussian random variable. (Proof of Proposition 3 is outlined in Appendix.)

\section{Lemmas}

\section{Lemma 1}

$$
\begin{aligned}
& \text { If } E_{2} \leq E_{1}<4 E_{2}, \\
& d_{\min _{1}}=d_{\min _{2}}=2\left(\sqrt{E_{1}}-\sqrt{E_{2}}\right)<2 \sqrt{E_{2}} \\
& d_{\min _{1}}=d_{\min 2}=\hat{d}_{\min 1}-\hat{d}_{\min 2}<\hat{d}_{\min 2}<\hat{d}_{\min 1}
\end{aligned}
$$

\section{Lemma 2}

$$
\begin{aligned}
& \text { If } E_{1}>4 E_{2}, \\
& d_{\min }=2\left(\sqrt{E_{1}}-\sqrt{E_{2}}\right)>d_{\text {min }_{2}}=2 \sqrt{E_{2}}
\end{aligned}
$$


$d_{\min 1}=\left(\hat{d}_{\min 1}-\hat{d}_{\min 2}\right)>d_{\min _{2}}=\hat{d}_{\min 2}$

\section{Lemma 3}

For the cross points where E1=4E2, the minimum distances satisfy the following equalities and inequality:

$$
\begin{aligned}
& d_{\text {min }_{1}}=d_{\text {min }_{2}}=2 \sqrt{E_{2}} \\
& d_{\min _{1}}=d_{\min _{2}}=\hat{d}_{\min 2}<\hat{d}_{\min 1}
\end{aligned}
$$

\section{Lemma 4}

If $\mathrm{E}_{2}=\alpha^{2} \mathrm{E}_{1}$, for $0 \leq \alpha \leq 1$, the minimum distances of the signal constellations can be written as follow:

\section{First signal constellation:}

$$
d_{\min }=2(1-\alpha) \times \sqrt{E_{1}}=(1-\alpha) \hat{d}_{\min 1}
$$

\section{Second Signal constellation:}

$$
\begin{aligned}
& \text { If } E_{1} \geq 4 E_{2} \text {, i.e., }\left(\alpha \leq \frac{1}{2}\right) \Rightarrow d_{\min _{2}}=\hat{d}_{\min 2}=\alpha \hat{d}_{\min 1} \\
& \text { If } E_{1}<4 E_{2} \text { i.e., }\left(\alpha>\frac{1}{2}\right) \Rightarrow d_{\min _{2}}=d_{\min 1}=(1-\alpha) \hat{d}_{\min 1}
\end{aligned}
$$

were $E_{2} / E_{1}=\alpha^{2}$, for some $\alpha \in[0,1]$.

\section{Lemma 5}

The BER for the strongest signal is given, when $E_{2}=\alpha_{2} E_{1}$, for $0 \leq \alpha \leq 1$, by :

$$
\begin{aligned}
& B E R_{1}=2 P(1-P) Q\left((1-\alpha) \sqrt{S N R_{1}}\right)+\left(P^{2}+(1-P)^{2}\right) Q\left((1+\alpha) \sqrt{S N R_{1}}\right) \\
& \underset{P_{P} / 2}{\longrightarrow} \frac{1}{2} \times Q\left((1-\alpha) \sqrt{S N R_{1}}\right)+\frac{1}{2} \times Q\left((1+\alpha) \sqrt{S N R_{1}}\right) .
\end{aligned}
$$

\section{RESULTS AND DISCUSSION}

Proposition 1 illustrates that when 2 signals are sent concurrently (scenario 2), the achievable minimum distance for any of the two signals' constellations is at most the difference of the achievable minimum distances if the 2 signals were sent separately (scenario 1). Clearly, sending the two signals simultaneously results into a lost of performance for the strongest signal, whereas the benefit is a better (double) efficiency of the bandwidth utilization.

From Proposition 2, if two signals with different energies are sent concurrently, the achievable minimum distances of the signals' constellations at the receivers are non-zero. Therefore, 
transmitting concurrent signals with different energies can allow multiplexing. Whereas when two signals with the same energy are sent concurrently, the signals' constellations at the receivers will both have a zero minimum distance, thus a very poor performance. This is, for example, the case of a concurrent transmission of 2 symmetrical, iid (independent and identically distributed), equal-powered signals, without time, frequency or code division.

Proposition 3 expressed the BER after the strongest signal detection as a function of the SNR. Since the first term in $6 \mathrm{~b}$ is mostly predominant, the BER depends strongly on the difference of the square root of the 2 SNRs. This is consistent with results from Propositions 1 and 2.

The result of lemma 1 shows that if the energies of two signals sent concurrently satisfies $\mathrm{E}_{2} \leq \mathrm{E}_{1}<4 \mathrm{E}_{2}$, the two signals will be detected with similar performance, which is lower than the achievable performances if they were transmitted separately. It can be seen as if each signal selflessly gives up part of its energy to combat the mutual interference so that both signals are detected with similar performance. However, signal 1 with higher energy has a higher contribution to fighting the mutual interference than the contribution of the weakest signal 2.

From Lemma 2 , if the energies of two signals sent concurrently satisfy $E_{1}>4 E_{2}$, the signal with the lowest energy does not contribute to fighting the mutual interference. This is a kind of selfish scenario where only the strongest signal 1 uses part of its energy to combat the interference caused to him by the second signal. The weakest signal uses all of its energy for its actual data transmission and will be detected with a performance similar to the case where it is transmitted separately with energy E2 (scenario 2).

Moreover, when the energies of two signals sent concurrently satisfy $E_{1}=4 E_{2}$, results (from Lemma 3) are similar to the previous (greedy) case where the strongest signal uses part of its energy to combat the interference caused by the second signal, whereas the weakest signal uses all of its energy for its data transmission. However, in this case, both signals end up having the same performance, equal to the performance of the weakest signal when sent separately (under scenario 1). In such a case, the total energy of both signals can be written as function of $d_{\text {min }}=$ $\mathrm{d}_{\min 1}=\mathrm{d}_{\min 2}$, as follows:

$$
E_{\text {total }}=1.25 \times d^{2} \text { min }
$$

For example, to double the minimum distance $d_{\min }$, up to 4 times the total energy would be required.

On the other hand, if the two signals were sent separately, the bandwidth efficiency would be twice smaller. However, the energy required to transmit each signal with a minimum distance $d_{\min }$ is $E_{1}=E_{2}=0.25 \mathrm{X} \mathrm{d}^{2} \min$. The total energy for a performance with $\mathrm{d}_{\min }=\mathrm{d}_{\min 1}=\mathrm{d}_{\min 2}$ at both receivers would then be :

$$
\mathrm{E}_{1}+\mathrm{E}_{2}=0.5 \mathrm{X} \mathrm{d}_{\text {min }}^{2}
$$

which is 2.5 time lower than the total energy for the same minimum distance if the signals were sent concurrently.

From Lemma 4, for $E_{2} \leq E_{1}$, the ratio $E_{2} / E_{1}$ can be written as $E_{2} / E_{1}=\alpha 2$, for some positive constant $\alpha$ less or equal to 1 . In that case, the minimum distance of the signal constellation at the receiver of the first (strongest) signal is a fraction of the minimum distance it would achieve if it was sent separately and decreases as $\alpha$ increases. Hence the closer the two energies are, the lower the minimum distance of the first signal constellation would be. For the second (weakest) signal, two 
cases can be considered. If $\alpha \leq 1 / 2$, then the (weakest) signal will be detected at the receiver with similar performance as if it was sent separately with Energy $E_{2}$. However, if $\alpha>1 / 2$, both signals will be detected with the same performance which is lower than both achievable individual performances if the signals were sent separately.

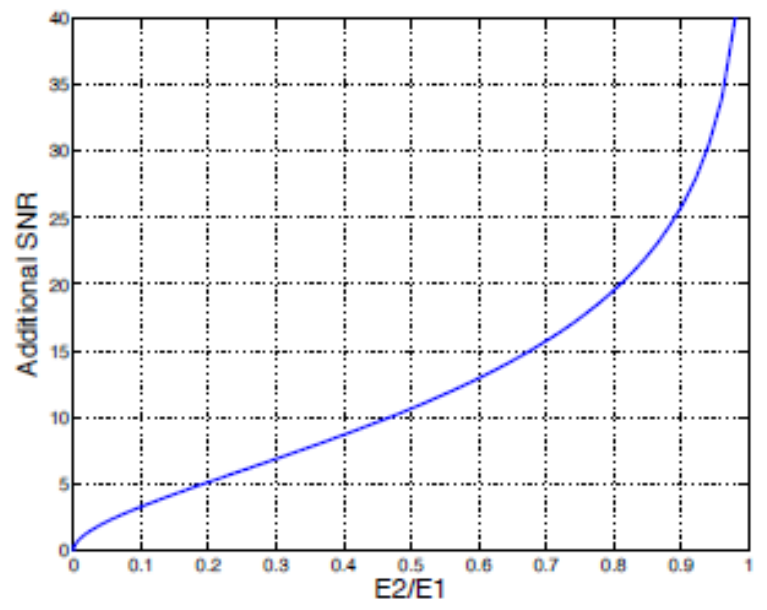

Figure 2: Additional SNR required for the first signal when sent concurrently with a second signal to achieve a performance similar to the case of separate transmission $\left(E_{2}=0\right)$ for uncoded transmission.

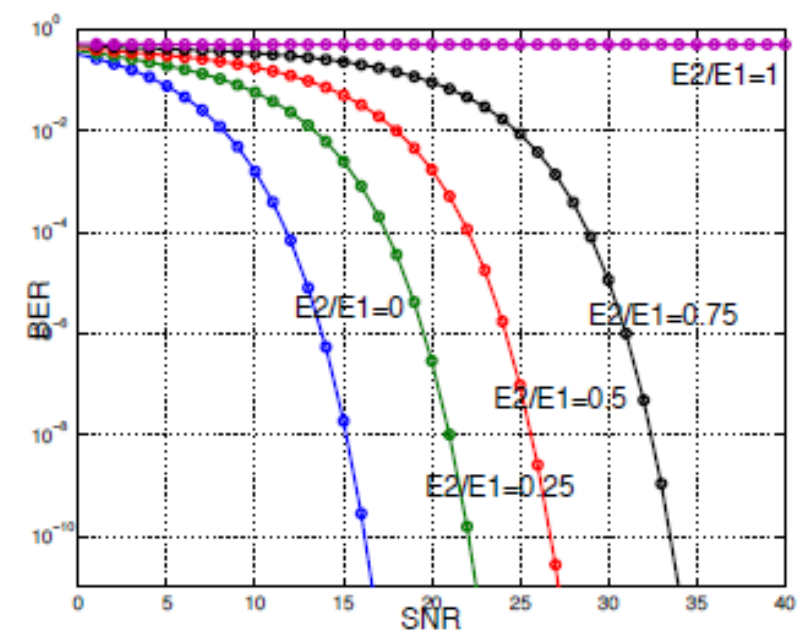

Figure 3: BER as function of SNR for the strongest signal with energy E1 when sent concurrently with another signal with energy $E_{2}$ for several values of $E_{2} / E_{1}$. The transmission is uncoded and uses BPSK modulation with $\mathrm{P}=1 / 2$.

Lemma 5 results from proposition 3 when the ratio $\mathrm{E}_{2} / \mathrm{E}_{1}$ equals $\alpha^{2}$. Figure 2 illustrates the additional SNR required for the strongest signal to achieve the same BER performance for uncoded transmission when it is sent concurrently with another signal. The additional SNR is in reference to the SNR it would require if it was sent separately in uncoded transmission with energy $E_{1}$. This additional SNR increases as $E_{2} / E_{1}$ increases. The additional SNR tends to infinity when $E_{2} / E_{1}=1$ and equals to 0 when $E_{2} / E_{1}=0$ (i.e., in the case of individual transmission, $E_{2}=0$ ). Figure 3 illustrates the (uncoded) BER as a function of the SNR for the strongest (first) signal when it is sent concurrently with a second signal for several values of $E_{2} / E_{1}$. The performance decreases as $E_{2} / E_{1}$ increases, where the case $E_{2} / E_{1}=0$ is equivalent to the case where the strongest signal is sent separately $\left(\mathrm{E}_{2}=0\right)$. For $\mathrm{E}_{2} / \mathrm{E}_{1}=1$, it is not possible to achieve a good performance for 
any value of the SNR. Note that the results in Figures 2 and 3 considered uncoded transmission and different results would be obtained if channel coding, multiplexing and/or further processing are assumed.

Finally, it is interesting to note that the system considered in this paper can also model a wireless transmission were at a given time instant, $\mathrm{a}$ and $\mathrm{b}$ are quasi-constant, known channel fading coefficients at the receivers.

\section{CONCLUSION}

This paper analyzes the performance of a transmission with two concurrent signals and trade-offs between the bandwidth and performance are discussed. More specifically, we present analytical expressions for the minimum distances for the two signals' constellations in various scenarios and selected BER performance results.

Our future work focuses on the design and analysis of transmision schemes for wireless systems when multiple signals are sent simultaneously and for the case of several cognitive signals transmission.

\section{REFERENCES}

[1] R. G. Gallager, "A perspective on multiaccess channels," IEEE Transactions on Information Theory, pp. 124 - 142, March 1985.

[2] T. M. Cover, J. A. Thomas, Elements of Information Theory, John Wiley \& Sons, New York, 1995.

[3] R. Etkin, D. Tse, and H. Wang, "Gaussian interference channel capacity to within one bit," IEEE Trans. Inf. Theory, vol. 54, no. 12, pp. 5534-5562, Dec. 2008.

[4] H. Sato, "The capacity of the Gaussian interference channel under strong interference," IEEE Trans. Inf. Theory, vol. IT-27, no. 6, pp. 786-788, Nov. 1981.

[5] A. A. Garba, J. Bajcsy, "Capacity of OCDMA network transmission with optical hard-limiters," Proc. Canadian Workshop on Information Theory, pp. 86 - 90, Montréal, QC, June 2005.

[6] G.J.M. Janssen, S. Ben Slimane, "Symbol Error Probability Analysis of a Mutiuser Detector for MPSK Signals Based on Successive Cancellation", IEEE J. Select. Areas on Communications, pp. 330- 338, February 2002.

[7] Tevfik Yucek, Huseyin Arslan, "A Survey of Spectrum Sensing Algorithms for Cognitive Radio Applications", IEEE Communication Surveys \& Tutorials, VOL. 11, NO. 1, pp. 116-130.

\section{APPENDIX}

\section{Proof of Lemmas}

Lemmas 1, 2 and 3, follow directly from Propositions 1 and 2. Lemma 4 results from proposition 2 , using $E_{2}=\alpha^{2} E_{1}$ and the fact that if the signals were sent separately with energies $E_{1}$ and $E_{2}$ respectively, the minimum distances for the signal constellations at the receivers would be:

$$
\hat{d}_{\min 1}=2 \sqrt{E_{1}} ; \hat{d}_{\min 2}=2 \sqrt{E_{2}}
$$

Lemma 5 is obtained from Proposition 3, by replacing $\mathrm{SNR}_{2}=\alpha^{2} \mathrm{SNR}_{1}$ in (6a) and (6b) when $\mathrm{E}_{2}=\alpha^{2} \mathrm{E}_{1}$ for $0 \leq \alpha \leq 1$.

\section{Proof of Proposition 3}

For the first signal, the BER can be approximated as follow: 


$$
\begin{aligned}
& B E R_{1} \leq P_{e}= P \times \operatorname{Pr}\left(Y<0 \mid X_{1}=a\right) \\
&+(1-P) \operatorname{Pr}\left(Y>0 \mid X_{1}=-a\right) \\
& \operatorname{Pr}\left(Y<0 \mid X_{1}=a\right)=P \times \operatorname{Pr}(n<-(a+b)) \\
&+(1-P) \times \operatorname{Pr}(n<-(a-b)) \\
&=P \times Q\left(\frac{a+b}{\sigma}\right)+(1-P) \times Q\left(\frac{a-b}{\sigma}\right)
\end{aligned}
$$

The first equality (13a) is obtained by using the decision regions illustrated in Figure 1 and the law of total probability. The last equality (13b) follows by using the definition of the Q-function for a Gaussian AWGN random variable with zero mean and variance $\sigma^{2}$. Similarly:

$$
\operatorname{Pr}\left(Y>0 \mid X_{1}=-a\right)=P Q\left(\frac{a-b}{\sigma}\right)+(1-P) Q\left(\frac{a+b}{\sigma}\right)
$$

Proposition 3 results by replacing expressions from equations (13b) and (13c) into (13a), using Equation (5), and replacing $\mathrm{SNR}_{1}=\mathrm{E}_{1} / \sigma^{2}$ and $\mathrm{SNR}_{2}=\mathrm{E}_{2} / \sigma^{2}$ in the previous expression. 